\title{
STRICT GEOMETRIC CALIBRATION OF AN UNDERWATER LASER TRIANGULATION SYSTEM
}

\author{
H. Sardemann*, C. Mulsow, H.-G. Maas \\ Institute of Photogrammetry and Remote Sensing, Technische Universität Dresden, Germany \\ (hannes.sardemann, christian.mulsow, hans-gerd.maas)@tu-dresden.de
}

Commission II, WG II/9

KEY WORDS: Triangulation, Laser line, Underwater Photogrammetry, Calibration

\begin{abstract}
:
This paper will describe a novel approach for the calibration of an underwater laser triangulation system. Underwater triangulation systems, consisting of a line laser and a camera can be used to determine the geometry of submerged objects or the topography of a water body bottom. Placing camera and laser line projector inside a waterproof housing leads to refraction effects at the air-glass-water interfaces, both of the laser light-sheet and image rays. This implies a deformed laser plane in the water and a curved line on the object surface. The proposed approach strictly models the geometry between camera, laser and housing. First experiments show, that the calibration method can be applied for water depth measurements with accuracies of $0.2-0.3 \mathrm{~mm}$ at depths in the order of $100 \mathrm{~mm}$.
\end{abstract}

\section{STATE OF THE ART}

Triangulation based measurement systems, consisting of a line laser and a camera, are commonly used for instance in industrial measurement tasks. By determining the location of the laser line in the image, a height profile along the laser line can be measured, resulting in a $2 \mathrm{D}$ measurement system. By adding movement of either the object or the triangulation sensor, 3D measurements can be performed. When used in the air, the laser light-sheet can be calibrated and modelled as a plane. Image measurements along the laser line are intersected with that plane in order to receive an object coordinate.

When laser triangulation is used in underwater environments, there are two main adaptions necessary: First, because of the increasing opaqueness of water for higher wavelengths (Hale and Querry, 1973), a green or blue laser is usually chosen. Furthermore, both the laser plane and the image rays are refracted at the air-housing and housing-water interfaces. Refraction influences have been considered with different approaches in the past. By using two separate housings for the camera and the laser, they can be arranged in a way, that the glass interfaces are planar and parallel to the image plane and perpendicular to the laser plane (e.g. Bleier et al., 2019). In that case, refraction has a radial symmetric distortion effect for the camera image and only results in a decrease of the opening angle for the laser plane. This setup enables the same measurement procedure as it is used in air, where the laser is parameterised as a plane and is intersected with image rays. However, placing camera and laser in different housings limits the compactness of the setup. Placing both the camera and the laser in the same housing, requires them to be oblique to obtain optimal intersection conditions. Palomer et al. (2018) presented a calibration method for a system, where the laser is arranged oblique to the interface. This leads to a curved laser in the water (Figure 1a, c). The authors parameterise the laser sheet as an elliptical cone, which can be intersected with image rays. However, Palomer et al. do not consider that the laser could also be rotated around a second axis, leading to an S-shaped laser sheet (Figure 1b,d). The approach proposed in this paper considers rotation of the laser around all axes.

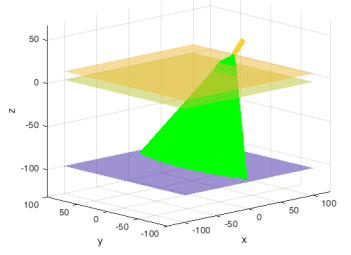

a

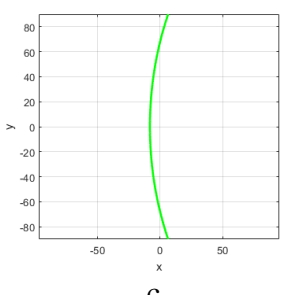

c

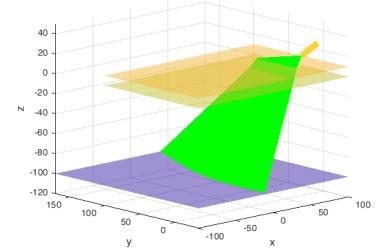

b

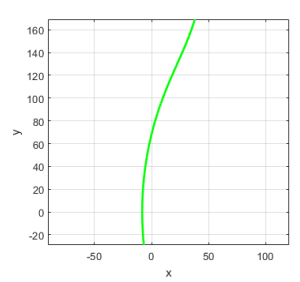

d
Figure 1: Deformation of a laser light-sheet penetrating from air through glass into water $(a, b)$ and their appearance on a planar surface (c, d). Rotated around the y-axis with $45^{\circ}$ (a-d) and additionally around the $\mathrm{x}$-axis with $30^{\circ}$ (only $\mathrm{b}, \mathrm{d}$ ).

\section{METHOD}

A triangulation system consisting of a line laser and a camera is used for underwater depth measurement. Both components are placed in the same housing with a transparent interface that is immersed into water. The laser light goes through the air, into the glass, from the glass into the water and hits the surface, where it is diffusely reflected. On its way back to the camera, it passes from water to glass to air into the camera. After measuring the laser line in the image (section 2.2), its image ray can be intersected with the deformed laser sheet in object space and a $3 \mathrm{D}$ coordinate can be determined (section 2.3).

\subsection{Set-Up}

Laser and image rays should optimally intersect orthogonally on the object in order to minimize the effect of inaccuracies in image

* Corresponding author 
measurements on object coordinates. This is accomplished by tilting laser and camera towards each other. An orthogonal setup of laser and camera in the air results in an angle smaller $90^{\circ}$ inside the water, since all rays are refracted towards the normal of the interface. Section 2.5 shows, how minimum and maximum measurable depth can be determined for every set-up. This can be utilized to determine the best set-up, i.e. adjusting the base between the sensors, the orientation of both sensors and the distance to the media interface, for a given depth range. Figure 3 shows an exemplary set-up of camera and laser and the resulting image.

\subsection{Line Detection}

First, the laser has to be detected in the image space. A typical set-up of laser and camera results in a curved line in the image either from top to bottom or from the left to the right side. Therefore, it can be measured row- or column-wise. The intensity of the laser decreases perpendicular to the line and can be described by a Gaussian curve. In reality, the laser shows a strong Speckle overlaying its Gaussian profile, complicating the determination of its peak.

A possible solution for a row- or column-wise detection of the line is to use least-squares-matching (LSM; Ackermann, 1984), with a parameterisation allowing only for scaling and translation in $\mathrm{x}$ or $\mathrm{y}$ direction. Using LSM enables subpixel accuracy precision. The matching-template consists of one or multiple Gaussian profiles. Its width has to be chosen considering the planarity of the measured surface. A wider patch, consisting of multiple profiles enables a stable measurement when the object surface is planar and the line is rather consistent. A rough surface leads to an uneven line, requiring a smaller patch with down to only one profile, resulting in a less precise determination of the line centre, being highly influenced by Speckle.

Figure 2 shows an example for the detection of a laser line that was projected on a planar surface. Speckle influence can be seen in Figure 2b. LSM was performed with a single-column-patch and a wider patch. The single column is one Gaussian curve (red line in Figure 2c or one column of Figure 2d), while the wide patch consists of 21 of these columns (Figure 2d). The result for a single-column patch is less consistent and precise.

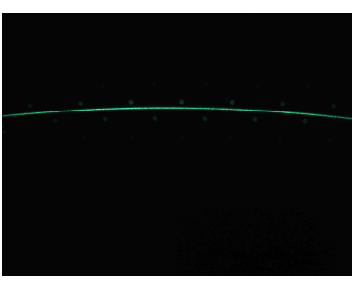

a

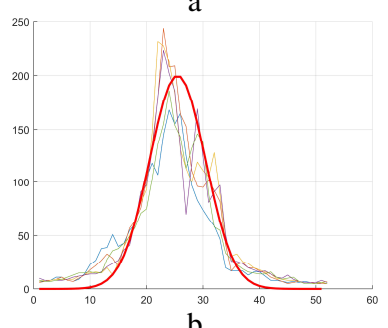

Figure 2: Laser line detection with LSM

(a): Laser Line observed through glass and water

(b): Detail of (a). Dots show LSM results with single-column patch (blue) and 21-column-wide patch (red)

(b): green-values of six columns in the centre of (b) (thin lines) and Gaussian profile used as single-column patch (bold red line) (c): patch with 21 Gaussian profiles

\subsection{Depth determination}

In order to measure the depth of a point along the laser line observed in the image, its image ray has to be calculated. When interior and exterior orientation of the camera are known, a 3D light ray can be modelled for every image observation. When the position, orientation and thickness of the media interface, i.e. housing, is also known, we can determine the ray path through the interface and determine its vector inside the water, using a 3D representation of Snell's law (a derivation can be found in Glassner, 1989):

$\vec{v}_{2}=\frac{n_{1}}{n_{2}} \vec{v}_{1}+\left(\frac{n_{1}}{n_{2}}\left(-\vec{N} \cdot \vec{v}_{1}\right)-\sqrt{1+\left(\frac{n_{1}}{n_{2}}\right)^{2}\left(\left(-\vec{N} \cdot \vec{v}_{1}\right)-1\right)}\right) \vec{N}$

where

$$
\begin{aligned}
& \vec{v}_{\{1,2\}}=\text { ray vectors in media } 1 \text { and } 2 \\
& n_{\{1,2\}}=\text { refraction indices of media } 1 \text { and } 2 \\
& \vec{N}=\text { normal of interface between the two media }
\end{aligned}
$$

The vector inside the water (red line in Figure 3) needs to be intersected with the laser light-sheet to receive a $3 \mathrm{D}$ position. The deformed laser sheet cannot be parameterized as a plane, but it can be described as a bundle of coplanar rays (sub-beams) with a perspective centre at the position of the laser diode. When exterior orientation and opening angle of the laser is known, its sub-beams can be determined and refracted through the glass into the water considering Eq. 1. There is only one sub-beam that intersects with the image ray (magenta line in Figure 3). This subbeam can be found iteratively by splitting the fan in two halves and choosing the one with the smaller distance between the central sub-beam and the image ray until it is underneath a threshold. The intersection determines the $3 \mathrm{D}$ position of the object surface. Figure 3 shows the image ray and its intersection with a specific sub beam of the laser sheet.

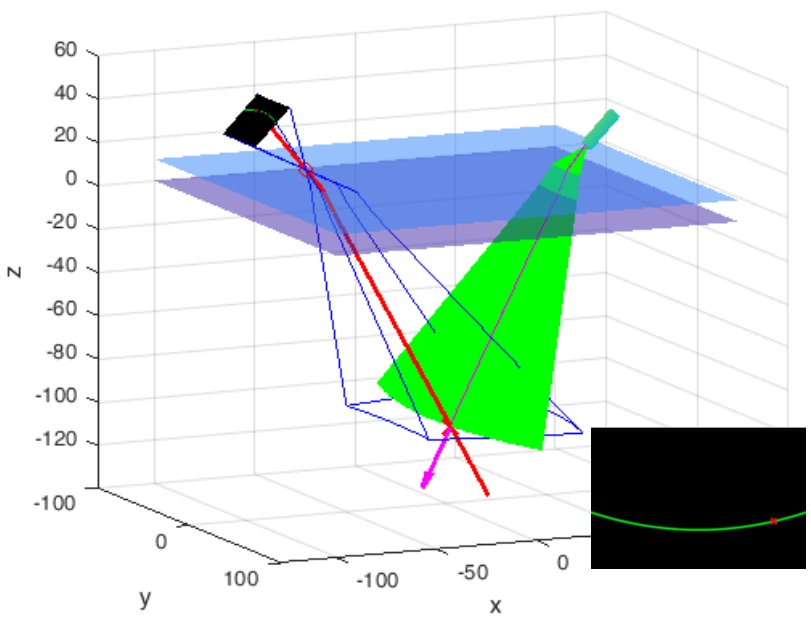

Figure 3: Intersection of image ray (red) and sub beam (magenta) of an image measurement on the line (small image). The two planes represent top and bottom of the glass interface between air and water.

\subsection{Calibration}

In order to enable 3D point measurements by intersecting image ray and laser sheet, exterior orientation of both the camera and the laser have to be calibrated relative to each other and relative to the housing interface. By measuring a bundle of sub beams, the laser sheet can be reconstructed. Further, a single sub beam can be determined by measuring at least two beam points. 
Practically this can be realised by capturing the projection of the laser sheet in two levels. The definition of a single laser beam can be reached, when constructing the surface nearest to the laser as a grid. Therefore, parts of the laser pass through the first level and hit the second level below. This creates a line pattern on both levels, where the corresponding endpoints span a vector representing a sub beam. A similar approach was used by Mulsow et al. (2006) for the indirect laser triangulation on reflective water surfaces.

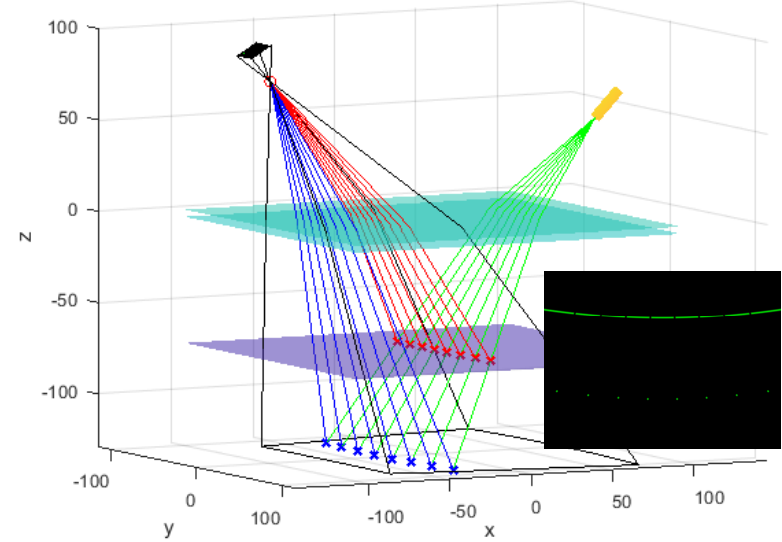

Figure 4: Calibration set-up. 3D-scheme and camera image. Sub-beams (green) are measured in two levels (red and blue crosses). The top level (purple plane) is designed as a grid. The image captured by the camera is shown in the small image.

Depending on the number of observed sub beams, camera position and orientation in relation to the glass surface, thickness and orientation of the glass, position and orientation of the two levels and refraction indices of air, glass and water can also be determined in a bundle adjustment using observations of the laser on two levels.

\subsection{Measurement Volume}

From a known or calibrated camera-laser set-up, the maximum achievable measurement volume can be determined. Therefore, the border pixels of the image are intersected with the laser sheet in order to get the outermost object coordinates that can be measured within the image (Figure 5).

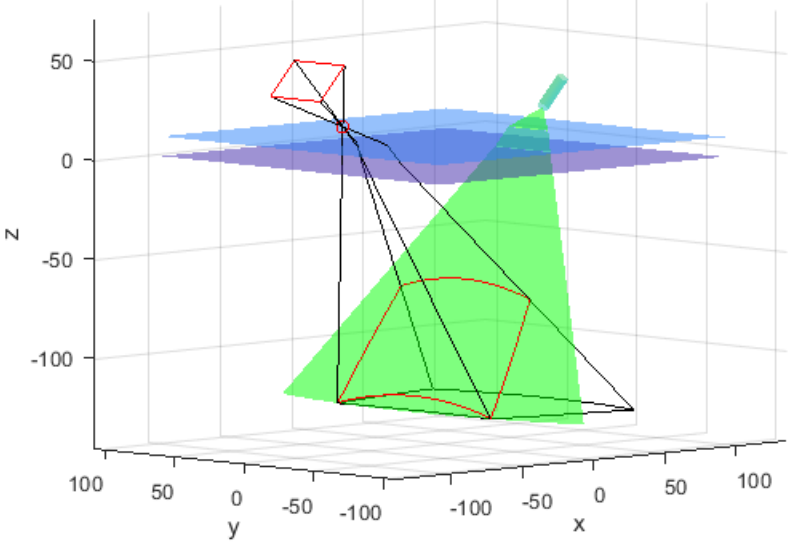

Figure 5: Measurement volume of a given camera-laser-set-up.

\section{EXPERIMENTS AND RESULTS}

For validation of the calibration procedure, a triangulation system, consisting of a green laser and an industrial camera has been placed in a glass housing. The laser has an opening angle of $90^{\circ}, 40 \mathrm{~mW}$ power and $520 \mathrm{~nm}$ wavelength. The camera has a $4 / 3$ sensor and a $20 \mathrm{~mm}$ lens. The glass housing was immersed into water. Figure 6 shows the experimental set-up

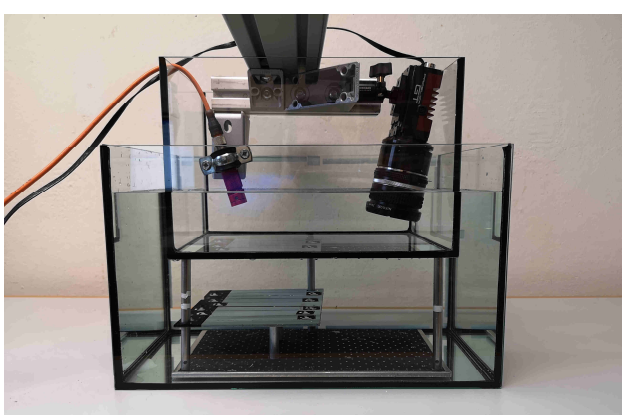

Figure 6: Set-up of the experiment, before camera and laser orientation were adjusted.

\subsubsection{Calibration}

For the calibration, two planes were placed in the field of view of camera and laser at 129 and $75 \mathrm{~mm}$ depth (Figure 7a). The upper plane is a grid, allowing for parts of the laser line to pass through. The distance of both planes relative to the glass interface and the thickness of the glass were measured with a calliper with $0.05 \mathrm{~mm}$ accuracy. Both planes and the interface are assumed planar and parallel. The camera's interior orientation was calibrated in advance by self-calibrating bundle adjustment, using a calibration panel consisting of coded markers and scalebars. For this experiment, the calibration described in section 2.4 was used to determine the position and orientation of both the camera and the laser relative to the glass interface, keeping all other parameters fixed. The laser line runs from the left to right image side and can be measured column-wise. Eight corresponding points were measured manually in the image on both levels (Figure 7b). Furthermore, 152 points along the line were measured using LSM (section 2.2) and included in the adjustment. The line points are constrained to lay on the according level.

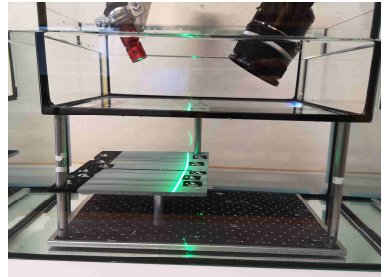

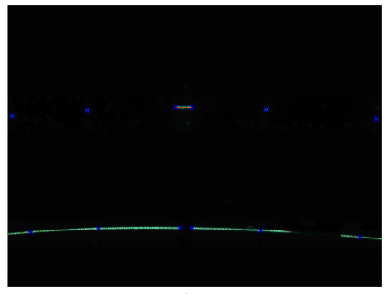

b
Figure 7: Calibration set-up (a) and image (b) with corresponding points on both levels (blue crosses) and additional points across the line (red dots).

Table 1 shows the results of this calibration. The origin of the coordinate system lies on top of the glass interface. The $\mathrm{x}$ - and $\mathrm{y}$ position of the laser have been fixed to zero, since only its height relative to the glass plane is relevant. One orientation angle of the laser (around the $\mathrm{x}$-axis) has also been set to zero, since it has the same effect that a wider opening angle would have.

Position of camera and laser were determined with standard deviations of $0.2-2 \mathrm{~mm}$, while the orientation angles were determined with standard deviations of $0.1^{\circ}-0.9^{\circ}$. This set-up with a $225 \mathrm{~mm}$ base and the orientations mentioned in Table 1 
enables a depth range of 54 to $185 \mathrm{~mm}$, following the concept of section 2.5 .

\begin{tabular}{|c|c|c|c|c|c|}
\hline Laser & value & Std.dev. & Camera & value & Std.dev. \\
\hline $\mathrm{x}$ & 0 & - & $\Delta \mathrm{x}$ & 220,554 & 2,334 \\
\hline $\mathrm{y}$ & 0 & - & $\Delta \mathrm{y}$ & 2,730 & 0,287 \\
\hline $\mathrm{z}$ & 106,26 & 0,856 & $\Delta \mathrm{z}$ & $-47,378$ & 2,463 \\
\hline$\omega$ & 0 & - & $\Delta \omega$ & 143,388 & 0,710 \\
\hline$\varphi$ & 31,324 & 0,871 & $\Delta \varphi$ & 33,409 & 0,911 \\
\hline$\kappa$ & 0,355 & 0,069 & $\Delta \kappa$ & $-67,311$ & 0,212 \\
\hline
\end{tabular}

Table 1: Calibration Results. Positions are given in $\mathrm{mm}$ and orientations as angles in degrees around the $x(\omega), y(\varphi)$ and $\mathrm{z}(\kappa)$ axes. Camera position and orientation is given relative to the laser.

\subsubsection{Measurements}

In order to test the achievable accuracy within the depth range, a planar panel was placed in three different water depths from 72 $122 \mathrm{~mm}$. The panel has circular markers on top of it, enabling a photogrammetric reference measurement. The reference measurement was performed in air, using a DSLR camera and additional coded markers and scale bars, resulting in reference depth values with $0.01-0.03 \mathrm{~mm}$ standard deviation (Figure 8). Camera and laser were mounted fix to each other and in reference to the glass interface for the calibration and the test depth measurements. The ground plate was $128.9 \mathrm{~mm}$ underneath the glass interface. The panel is $6.2 \mathrm{~mm}$ thick, resulting in a reference depth of $122.7 \mathrm{~mm}$, when it is placed directly on top of the ground plate. It was placed on two more height levels for this test.

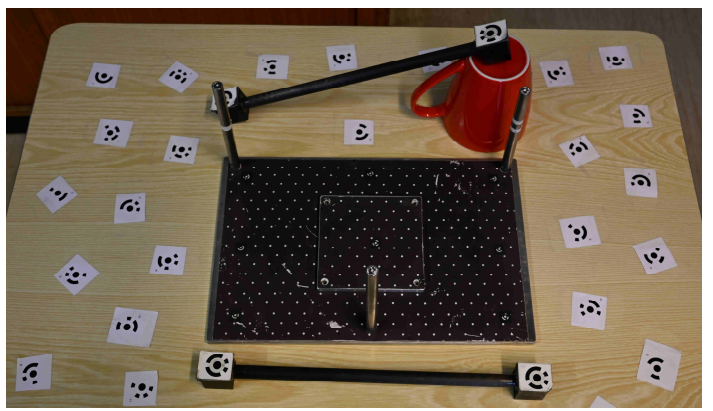

Figure 8: Measurement object consisting of ground plate and measurement panel.

Figure 9 shows the camera image for $128.9 \mathrm{~mm}$ and $122.7 \mathrm{~mm}$ depth, with the laser points that were determined column-wise by LSM. Those image points were intersected with the laser sheet, resulting in $3 \mathrm{D}$ object points. It also shows the epipolar lines for both reference depths. The mean distance between the measured points and the reference depths is $0.03 \mathrm{~mm}$ with $0.21 \mathrm{~mm}$ standard deviation. Figure 10 shows the distance between measurement and reference in object space.

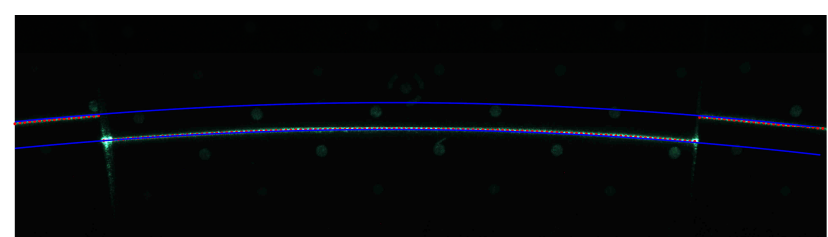

Figure 9: Laser line measurement in the image (detail) with LSM-based image measurements (red dots) and epipolar lines of the reference depths (blue lines).

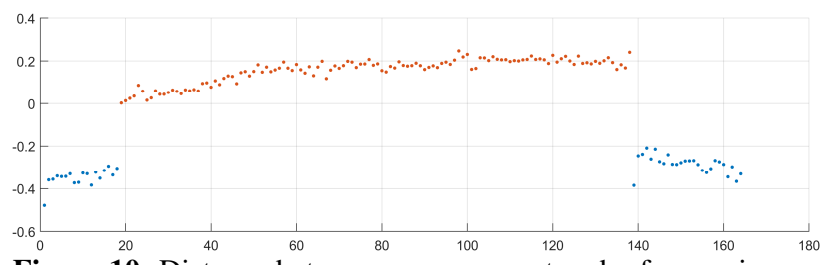

Figure 10: Distance between measurement and reference in $\mathrm{mm}$ for $128.9 \mathrm{~mm}$ (blue) and $122.7 \mathrm{~mm}$ depth (red).

The panel was also placed at $72.7 \mathrm{~mm}$ and $83.1 \mathrm{~mm}$ depths resulting in mean errors of 0.07 and $0.46 \mathrm{~mm}$ with standard deviations of 0.15 and $0.34 \mathrm{~mm}$.

\section{SUMMARY AND OUTLOOK}

This paper presents a novel geometric modelling and calibration method for an underwater laser triangulation system. The calibration of the exterior orientations of camera and laser can be achieved with only one image of a calibration object, that consist of two planes, where the top plane is a grid that allows parts of the laser to pass through. First experiments show, that the concept can be applied. Nevertheless, it can be observed, that there are systematic errors left in the results. This can be caused by different influences that will be examined in future work. One possible explanation is the choice of the refraction indices. The exact value for the used glass was unknown. It should be possible to include the refraction index of the glass interface into the adjustment, enough input measurements are available. Another influence was the gridded panel that was used as the top level for calibration. It was not very solid and might have bended, resulting in inaccurate calibration results. A new calibration grid has already been designed and 3D-printed and will be used for upcoming experiments. Another option is to stabilize the calibration by adding a third level to the process.

\section{ACKNOWLEDGEMENTS}

Funded by the Deutsche Forschungsgemeinschaft (DFG, German Research Foundation) - 433522300 .

\section{REFERENCES}

Ackermann, F., 1984: Digital Image Correlation: Performance and Potential Application in Photogrammetry. Photogrammetric Record, 11(64), 429-439

Bleier, M., van der Lucht, J., Nüchter, A., 2019: Towards an Underwater 3D Laser Scanning System for Mobile Mapping. Proceedings of the IEEE ICRA Workshop on Underwater Robotic Perception (ICRAURP '19). Montreal, Canada

Glassner, A. S., 1989: Surface Physics for Ray Tracing. In Glassner, Andrew S. (Ed.): An introduction to ray tracing. Academic Press. 121-160.

Hale, G. M., Querry, M. R. (1973): Optical Constants of Water in the 200-nm to 200- $\mu \mathrm{m}$ Wavelength Region. Applied Optics, 12(3), 555.

Mulsow, C., Schulze, M., Westfeld, P., 2006: An optical triangulation method for height measurements on non-stationary water surfaces. Int. Arch. Photogramm. Remote Sens. Spatial Inf. Sci., Vol. XXXVI, Part 5, 213-217.

Palomer, A., Ridao, P., Youakim, D., Ribas, D., Forest, J., \& Petillot, Y., 2018: 3D laser scanner for underwater manipulation. Sensors, 18(4), 1086. 